\title{
Establishing Amyloid PET Imaging Biomarkers: Ongoing Efforts
}

$\mathbf{T}$ he concept of imaging biomarkers was conceived in the late 1990s. The process of deduction from "complex" imaging data to a few simpler indices is often required for biomarkers to be quantitative and reproducible. Widespread examples in the field of PET imaging include the standard uptake value (SUV) to represent tumor FDG uptake ${ }^{1}$ and quantitative statistical mapping methods by using the $z$ scores applied to brain FDG PET scans. ${ }^{2}$ Such methods have been used for the diagnosis and therapeutic evaluation of various medical conditions. Now, clinically available amyloid PET tracers permit the development of such approaches for amyloid PET interpretation.

The study by Nayate et $\mathrm{al}^{3}$ used 60 cases of amyloid PET scans (30 scans as test cases) obtained from the Alzheimer Disease Neuroimaging Initiative (http://www.adni-info.org). They calculated the standard uptake value ratio (SUVr) (cortical regions to whole cerebellar uptake) by using software available on a workstation and presented SUVr values to readers who were interpreting amyloid PET scans. They found that interreader agreement increased significantly when the scans were presented with SUVr information. This study illustrates nicely that quantitative biomarker information might improve scan interpretation across different readers with different degrees of experience in amyloid PET interpretation.

The group from Pittsburgh conducted the seminal work on the quantitative assessment of imaging biomarkers for amyloid PET. Lopresti et $\mathrm{al}^{4}$ compared more rigorous quantitative makers, such as distribution volume, that were obtained by using dynamic PET imaging and Logan graphical analysis. They found that simpler indices, such as SUVr, produced accuracy comparable with more elaborate methods in distinguishing scans of Alzheimer disease versus control conditions. ${ }^{4}$ One major advantage of SUVr is that it can be estimated on a single static PET scan without requiring dynamic imaging or complex tracer analysis. This simplicity is well-suited in clinical applications. Although the term "SUVr" has been used frequently in the field of amyloid PET, SUVr merely represents a ratio of radiotracer uptake of a target region (ie, cortical regions) divided by a reference region (ie, the cerebellum). Pixel counts on the reconstructed amyloid PET images do not need to be converted to SUV values before the calculation of SUVr. It is equivalent to a "target-to-background" ratiothe index that has been used in nuclear medicine for many years.

While imaging biomarkers such as SUVr for amyloid PET can provide information complementary to qualitative scan interpretation, it is important to know that SUVr values can be affected by various factors. For example, different amyloid tracers ( 3 tracers are currently approved by the US FDA) can produce different SUVr values. ${ }^{5}$ Scan timing, size of ROIs, and image reconstruction methods can produce different SUVr values from the same patient, similar to SUV values used for FDG PET in cancer work-up. Different reference regions can produce substantially different SUV values (such as whole cerebellum versus cerebellar gray matter versus cerebral white matter). Various investigators have explored the best reference region for amyloid PET analysis. Because of the method- and tracer-dependent nature of the SUVr, the same SUVr threshold for distinguishing scans with positive-versus-negative findings cannot be applied to scans obtained with different tracers, different imaging methods, and different ROIs. There has been an ongoing effort to standardize quantitative biomarkers for amyloid PET through the Quantitative Imaging Biomarkers Alliance of the Radiological Society of North America (http:// www.rsna.org/QIBA). A method to unify amyloid PET analysis for different radiotracers has also been proposed. ${ }^{6}$

One critical question that was not addressed in the current study by Nayate et $\mathrm{al}^{3}$ is the accuracy of scan interpretation by using SUVr information. Presentation of the SUVr values at the time of scan interpretation improved interreader agreement. Was the diagnostic accuracy also improved with the SUVr, or did readers consistently make wrong diagnoses on certain cases because of the SUVr values? Without a gold standard (which typically requires postmortem data), the current study by Nayate et al cannot assess such critical questions related to the accuracy of scan interpretation. When reading amyloid PET scans in the clinic, we occasionally encounter cases that have discrepant impressions from qualitative visual interpretation and quantitative SUVr assessment. In such cases, 
which one should be trusted? This problem requires more robust prospective validation and standardization of amyloid PET biomarkers. Such effort is currently underway in molecular brain imaging communities.

\section{REFERENCES}

1. Wahl RL, Zasadny K, Helvie M, et al. Metabolic monitoring of breast cancer chemohormonotherapy using positron emission tomography: initial evaluation. J Clin Oncol 1993;11:2101-11

2. Minoshima S, Frey KA, Koeppe RA, et al. A diagnostic approach in Alzheimer's disease using three-dimensional stereotactic surface projections of fluorine-18-FDG PET. J Nucl Med 1995;36: $1238-48$

3. Nayate AP, Dubroff JG, Schmitt JE, et al; Alzheimer's Disease Neuroimaging Initiative. Use of standardized uptake value ratios de- creases interreader variability of $[18 \mathrm{~F}]$ florbetapir PET brain scan interpretation. AJNR Am J Neuroradiol 2015;36:1237-44

4. Lopresti BJ, Klunk WE, Mathis CA, et al. Simplified quantification of Pittsburgh Compound B amyloid imaging PET studies: a comparative analysis. J Nucl Med 2005;46:1959-72

5. Landau SM, Thomas BA, Thurfjell L, et al; Alzheimer's Disease Neuroimaging Initiative. Amyloid PET imaging in Alzheimer's disease: a comparison of three radiotracers. Eur J Nucl Med Mol Imaging 2014;41:1398-407

6. Klunk WE, Koeppe RA, Price JC, et al. The Centiloid project: standardizing quantitative amyloid plaque estimation by PET. Alzheimers Dement 2015;11:1-15.e1-4

S. Minoshima

Department of Radiology University of Utah Salt Lake City, Utah

http://dx.doi.org/10.3174/ajnr.A4433 